\title{
CMLBPINCOHERENT: A NEW CONTEXTUAL IMAGE DESCRIPTOR FOR SCENE CLASSIFICATION
}

\author{
Matheus Vieira Lessa Ribeiro*, Evandro Ottoni Teatini Salles* \\ * Av. Fernando Ferrari, 514 \\ Universidade Federal do Espírito Santo \\ Vitória, ES, Brazil
}

Emails: matheus.v.ribeiro@aluno.ufes.br, evandro@ele.ufes.br

\begin{abstract}
Scene classification has been widely studied in the last years and is a significant computer vision problem. Currently, this application has been involved by several researchers, since the creation of robust datasets until the utilization of high-level techniques with great performance. However, due to the high complexity and variability from scenes, there is still so much to be studied and implemented aiming to improve better results. This paper proposes a new visual descriptor for scene classification, the CMLBPIncoherent, which models the distribution of local structures through Local Binary Pattern (LBP) algorithm combined with contextual information, and discards homogeneous regions by Color Coherent Vector (CCV) algorithm. The proposal was tested using some traditional scene databases and the results illustrate a better performance in comparison with other visual descriptors known in the literature.
\end{abstract}

Keywords - Local Binary Pattern, Digital Image Processing, Scene Classification, Computer Vision.

Resumo- A classificação de cenas tem sido amplamente estudada nos ultimos anos e é um problema considerável em visão computacional. Atualmente, esta aplicação tem sido abrangida por muitos cientistas, desde a criação de banco de dados robustos até a utilização de técnicas de alto nível com alta performance. Entretanto, devido à complexidade e variabilidade das cenas, há ainda muito a ser estudado e implementado com o objetivo de melhorar os resultados. Este artigo propõe um novo descritor visual para a classificação de cenas, o CMLBPIncoherent, que modela a distribuição das estruturas locais através do algoritmo Local Binary Pattern (LBP) combinado com informação contextual, e descarta regiões homogêneas pelo algoritmo Color Coherent Vector $(\mathrm{CCV})$. A proposta foi testada usando alguns banco de dados tradicionais e os resultados ilustram uma performance melhor em comparação com outros descritores visuais conhecidos na literatura.

Palavras-chave - Local Binary Pattern, Processamento Digital de Imagens, Classificação de Cenas, Visão Computacional

\section{Introduction}

Scene classification has been an important and challenging task in the field of robotics and computer vision. There are many applications in this area since content-based image retrieval until autonomous navigation by robots. The goal of image scene classification is to automatically assign a semantic category to each given image based on some predefined knowledge (Bian et al., 2017).

In literature, we find several algorithms from different complex level and approach. However, despite the advances and the great effort in image scene classification, it is still faced with many problems. The main reason is the high scene variability. A single class of scene might suffer from variations of illumination, scale, occlusions and viewpoints, making this topic a challenging task (Shi et al., 2015).

Although those difficulties do not embarrass humans during a scene recognition, that can identify a significant amount of information in a glance, for a machine this task could be arduous. Hence, researches based on how humans perceive a scene image have been made since the last decades to inspire new computational algorithms (Jiang et al., 2010) (Potter, 1975).

Through these studies, Oliva and Torralba (2001) proposed a holistic algorithm based on some perceptual dimensions of the scene. From these dimensions, a multidimensional space is generated, and the scenes with the same properties are clustered. These dimensions are called as the gist of the scene, it is the meaningful information that a person captured by a glance.

The holistic approaches describe an entire image as a single object and extract their main characteristics. These approaches are a standard method for scene classification, they are ideals when the scene has low variations and information. However, the outcome gets worse for complex images, with many objects, for example (Chen et al., 2015).

In fact, for complex images is more appropriate to divide an image in grids to extract the mainly local features from each grid, the local approaches (Li and Fei-Fei, 2007). An example of this method is the bag-of-visual-words (BoVW) model, which the image is represented by a set of visual words and their histogram of occurrences(Yang et al., 2007). The visual words are established by a clustering process. However, this technique requires a high computational cost (Shahriari and Bergevin, 2017).

Therefore, for a scene classification task, different feature descriptors can be used for distinct classes. For example, the gist is appropriated for flat scenes composed by semantic class as the sky, 
water, and sand, whereas BoVW is appropriated for indoor classes with many random directions borders and objects as the bedroom, bathroom, and library. Currently, many researchers are joining both approaches to construct a vector with more descriptive power and more robust to achieve better results.(Bian et al., 2017).

Fan (Fan, 2016) has proved that the spatial context information from the scene configuration is one of the critical factors for the scene classification task. Chu and Zhao (2014) utilized contextual information from local features generated by SIFT to improve scene classification in available datasets. Shahriari and Bergevin (2017) explored the contribution of the contextual information in homogeneous regions, and the authors concluded that this information improves the results.

This paper proposes the CMLBPIncoherent as a new visual descriptor involving contextual information and local structures detected from Local Binary Pattern (LBP) (Ojala et al., 1996). As will be exposed, CMLBPIncoherent discards homogeneous regions, it was inspired in the Color Coherent Vector algorithm (CCV)(Pass et al., 1997). This approach has some advantages: few parameters to tune, simple implementation and low computational cost.

The outline of this article is structured as follows: in Section 2 a description of Local Binary Pattern, Contextual Modified Census Transform, and Color Coherent Vector is approached, Section 3 describes the proposed method CMLBPIncoherent, Section 4 presents the experimental results and in Section 5 our conclusions and future investigations.

\section{Technical Background}

\subsection{Local Binary Pattern}

Local Binary Pattern is a typical feature descriptor with high power discriminator and low computational cost. This fact explains why it has been used in many applications for digital image processing such as texture classification (TURAN et al., 2018), hands gestures classification (Tabatabaei and Chalechale, 2018) and face recognition (Salyut and Kurnaz, 2018).

LBP labels the pixels of an image by thresholding the neighborhood of each pixel in a clockwise way and considers the final result as a binary number with eight digits. This method is illumination invariant and grayscale invariant. Figure 1 shows this process.

The equation of the final value for LBP algorithm in the center pixel follows as:

$$
L B P(x, y)=\sum_{n=0}^{7} s\left(i_{n}-i_{c}\right) * 2^{n}
$$

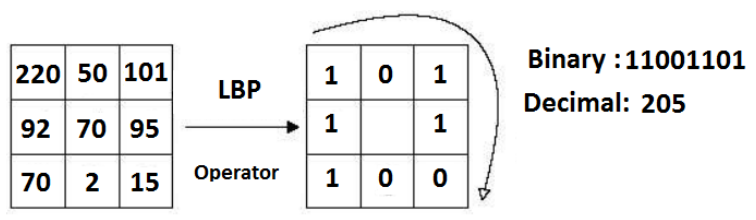

Figure 1: Local Binary Pattern.

where $x$ and $y$ are the coordinates from center pixel in original image, $i_{c}$ is the graylevel of center pixel and $i_{n}$ is the graylevel of its neighborhood pixels. The $s(z)$ function is defined as 0 for $z<0$ and 1 otherwise.

The final descriptive vector is the histogram of the LBP image.Wu and Rehg (2011) created the Centrist, a histogram of Census Transform (CT), equivalent to the LBP code, but taking a different bit ordering.

\subsection{Modified Census Transform}

In some cases, the LBP does not capture the correct local structure, mainly when the center pixel has a high value. To overcoming this shortcoming, Zabih and Woodfill (1994) proposed the Modified Census Transform (MCT), which differs from LBP in the comparison of the center pixel. MCT compares the eight neighborhood pixels and the center pixel with the mean of their intensity value, therefore, the final value ranges from 0 to 511 . de Souza Gazolli and Teatini Salles (2014) extended the MCT8, which the center pixel is not compared, the final value ranges from 0 to 255 . The MCT8 equation is as follows:

$$
\operatorname{MCT} 8(x, y)=\sum_{n=0}^{7} s\left(i_{n}-i_{m}\right) 2^{n}
$$

where $i_{m}$ is the mean of the $3 \times 3$ window of pixels. The Figure 2 shows the final result of LBP and MCT8 algorithm. According to equations 1 e 2, the black pixel has value 0 and white pixel has value 1 . The MCT8 described the relation between pixels better than LBP in this example. That is why the value of the center pixel from both algorithms was distinct, while for LBP the center pixel is its absolute value, for MCT8 the center pixel is the sample mean value of $3 \times 3$ window pixels.
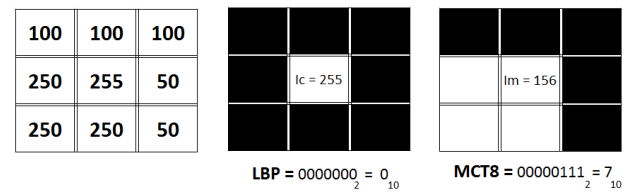

Figure 2: LBP and MCT8 algorithm. 


\subsection{Contextual Mean Census Transform}

To improve the image representation, de Souza Gazolli and Teatini Salles (2014) proposed the Contextual Mean Census Transform (CMCT). The CMCT utilizes contextual information from local structures. This idea is motivated by pixels with similar local structures but with differences in their neighborhood. For scene classification, e.g, a white pixel might be a snow, but its environment is composed of blue pixels, we would suppose that it must be a cloud.

The CMCT process is described as follows: firstly MCT8 is computed over the original image, then a MCT8 image and its histogram are generated. Finally, we apply again the MCT8 algorithm over the MCT8 image, the new image and its histogram are computed. The final descriptor vector is constructed by the concatenation of both histograms computed.

The Figure 3 shows the result of the MCT8 when the center pixel has two different contextual information. Note that for different contextual structures the MCT8 values are equals because MCT8 compares only the eight neighborhoods from the center pixel and ignores the contextual information. Nonetheless, when we set MCT8 again, the contextual information becomes relevant and the final value is distinct in both cases.

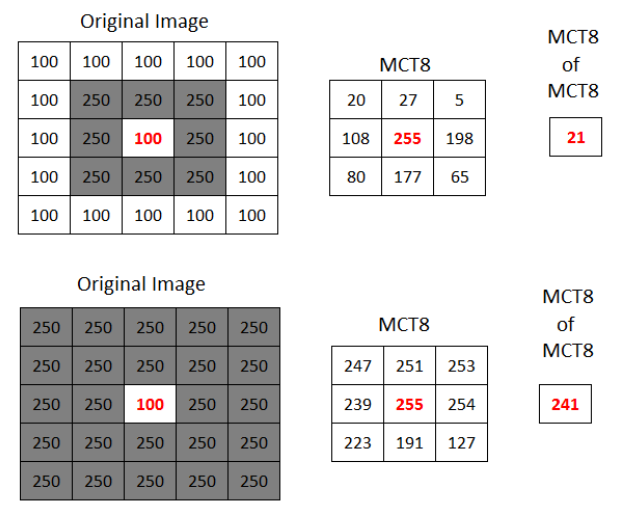

Figure 3: Comparison between MCT8 and CMCT values with different neighborhoods.

\subsection{Color Coherent Vectors}

Based on the advantages and efficiency that color histogram offers to describe an image in many applications, Pass et al. (1997) proposed the Color Coherent Vector (CCV). This algorithm is motivated by the fact that color histograms do not have spatial information. In other words, different images might have the same number of pixels of a specific color, independently whether they are clustered or spread among the image.

Firstly, the colored image is transformed into a grayscale image and the color space is discretized in buckets, such that the image contains lower distinct values. Therefore, the bucket 0 contains the intensities 0 through 9 , bucket 1 contains 10 through 19 and so on, in our work, we vary the length of the bucket, as it will be described.

Secondly, the pixels from the discretized image are classified into two classes: coherent or incoherent. The coherent pixel is part of a group of pixels with the same color connected between them, whereas incoherent pixel does not have pixels with its same value in the neighborhood. The size of the coherent group must be above a threshold chosen by the user.

\subsection{Support Vector Machines}

Support Vector Machines (SVM) is a supervised learning algorithm which tries to find an optimal hyperplane able to separated two classes such that the expected classification error is minimized (Burges, 1998). The solution to this optimization can be easily found if the data are linearly separable. However, this task might be complicated when the data are not linearly separated.

When it occurs, the data is transformed into a higher dimensional where the linearity is achieved. This transformation can be made through a kernel function which maps the data space in a higher dimensional space linearly separable. This method also defines a security region, called margin, around the separation hyperplane (Burges, 1998).

In literature, many articles adopting the SVM classifier have been published in the field of scene classification, independently of complexity of approach and descriptive features. Therefore, SVM becomes one of the most important classifiers in the scene classification (Mandhala et al., 2014).

\section{Contextual Mean Local Binary Pattern Incoherent}

The Contextual Mean Local Binary Pattern Incoherent (CMLBPIncoherent) integrates contextual and spatial information with local structures achieved by LBP and CMCT. Firstly, LBP is applied in the original image. Then, the MCT8 is applied two consecutive times in the original image, we call the generated image from this process of MCT8ofMCT8 image. Finally, the histograms of incoherent pixels from LBP image and MCT8ofMCT8 image are computed and concatenated to construct the final visual descriptor vector of the original image. The flowchart of the proposed method is represented in the Figure 4.

The values generated by LBP image and MCT8ofMCT8 image are quantized in buckets of 1 and 2 levels of intensity, generating images of 256 and 128 graylevels respectively. Posteriorly, the pixels are separated in coherent and incoherent groups, and the histograms are calculated. 


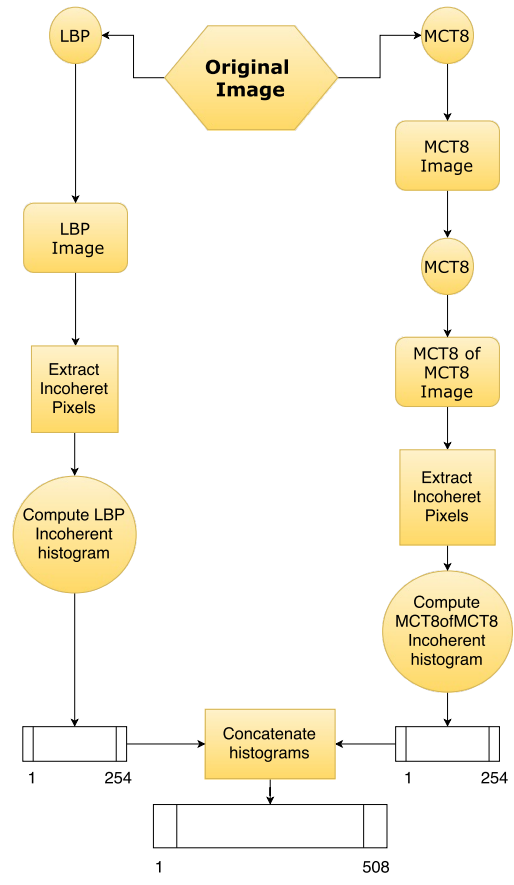

Figure 4: CMLBP Incoherent flowchart.

The Figure 5 shows the distribution of coherent pixels for LBP, MCT8 and MCT8ofMCT8 images. When we apply the Coherent/Incoherent algorithm in LBP image, the coherent pixels appear in homogeneous regions and uniform texture regions. Applying MCT8 in the same scene, we observe an increase in the amount of coherent pixels in the image, as the wall of the house in Figure 5. It occurs because MCT8 captures more homogeneous regions than LBP, as the MCT8 utilizes the mean operator.
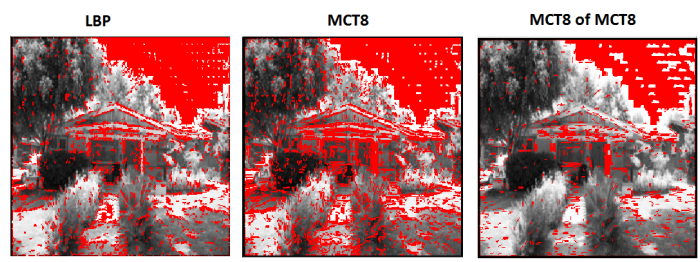

Figure 5: Coherent pixel (red pixels) in LBP, MCT8 and MCT8ofMCT8 image.

Nevertheless, the MCT8ofMCT8 also captures coherent pixels in homogeneous regions, but with a lower amount than the MCT8 image. It happens because MCT8ofMCT8 adds contextual information, so the coherent pixels is founded in regions with a high degree of homogeneity.

Therefore, although LBP and MCT8ofMCT8 capture homogeneous regions, the LBP Coherent captures uniform texture regions whereas MCT8ofMCT8 does not. Note that, in the Figure 5, LBP Coherent captures pixels in homogeneous regions and homogeneous texture regions such as the grass, while the MCT8ofMCT8 Coherent cap- tures pixels in regions with a high degree of homogeneity such as the sky and the wall.

\section{Experiments and Discussion}

In this section we investigated, over the available datasets, the accurate of our method and compared it with existing works.

\subsection{Datasets and Setup}

Our descriptor has been applied on four datasets, which including indoor and outdoor scenes, with many or few objects.

- 8-category scenes - provided by Oliva and Torralba (2001). This dataset contains 2,688 color images, with size 256 x 256 into eight categories: coast (360 images), forest (328 images), mountain (274 images), open country (410 images), highway (260 images), inside city (308 images), tall building (356 images) and street (292 images).

- 15-category scenes - provided by Lazebnik et al. (2006). The 8 scenes from the previous dataset compose this dataset, more 7 scenes ( 5 scenes provided by the Fei-Fei and Perona (2005) and 2 scenes provided by the author: bedroom (216 images), kitchen (210 images), living room (289 images), office(215 images), suburb (241 images), industrial (311 images) and store (315 images). This dataset contains 4,486 gray-values images in total. The mean size of all images is approximately $300 \times 250$.

- 8-class sports events - provided by Li and Fei-Fei (2007). This dataset contains 1,579 images of eight sports: badminton (200 images), bocce (137 images), croquet (236 images), polo (182 images), rock climbing (194 images), rowing (250 images), sailing (190 images) and snowboarding (190 images).

- 67-class indoor scene - provided by Quattoni and Torralba (2009). This dataset contains 15,616 images with many indoor images and great variability of each class. Besides, the number of images per class and the image size varies. This dataset poses a challenging classification problem (Quattoni and Torralba, 2009).

For the experiments, each category in a dataset was split randomly into a training set and a test set. The random splitting was repeated five times, and the average accuracy was reported, as adopted by Oliva and Torralba (2001). For the SVM, we employed a Gaussian kernel with gamma factor equals to 3 . The $\mathrm{C}$ parameter, which controls the SVM errors during training and margin maximization, was fixed after tests equal to 13 . 
For the 8-category scenes and 15-category scenes, 100 images in each category were used for training, and the remaining images constituted the testing set as proposed by Oliva and Torralba (2001). In 8 sports events categories, we used 70 images per class for training and 60 for testing, as in Li and Fei-Fei (2007). Following Quattoni and Torralba (2009), in the 67-class indoor dataset, we used 80 images in each category for training and 20 images for testing.

The presented results hereinafter were carried out in the software environment Matlab. For SVM classifier we utilized LIBSVM, a Library for Support Vector Machines (Chang and Lin, 2011). The strategy adopted for multi-class classification was one-against-all.

All color images were converted to grayscale, and the images were resized. The new size of an image with dimension $[\mathrm{a} \times \mathrm{b}]$ was:

$$
\begin{array}{r}
a_{n}=k \\
b_{n}=\frac{b * k}{a},
\end{array}
$$

where $\left[\begin{array}{lll}a_{n} & \mathrm{x} & b_{n}\end{array}\right]$ is the new size of the image, and $k$ is the new value of the width chosen. In our paper, we set $k=240$ to approximate with 8 scenes dataset dimension.

We normalize the histograms such that they have zero mean and unit norm, moreover, we remove the two extreme bins ( 0 e 255) in all histograms, as proposed in Centrist (Wu and Rehg, 2011).

\subsection{Size of Coherent Regions}

The next step was to set the region size to classify the pixels between coherent and incoherent, we called this term as size coherent threshold (SCT). In Pass et al. (1997), for example, the SCT for CCV was $1 \%$ of image size and in (Vailaya et al., 1998) the threshold for EDCV was $0,1 \%$. Therefore, the value of this threshold relies into the features required.

The Figure 6 presents the outcomes for some thresholds in the 15-category scenes dataset when we used the LBP image. The Figure contains outcomes for the joined histograms(Coherent and Incoherent) and individually separated.

Observe that, for $1 \%$ threshold value the recognize made by joined histogram has a bigger value than $0.1 \%$ threshold. It happens because $1 \%$ of an image means approximately 600 pixels, a high number for connected pixels group with the same value, that is why there are few coherent pixels in the image. So, the outcome carried from this threshold is due to the high contribution from Incoherent histogram and low contribution for Coherent histogram.

If the SCT is reduced by $0.1 \%$, the amount of coherent regions slightly increases, it causes more

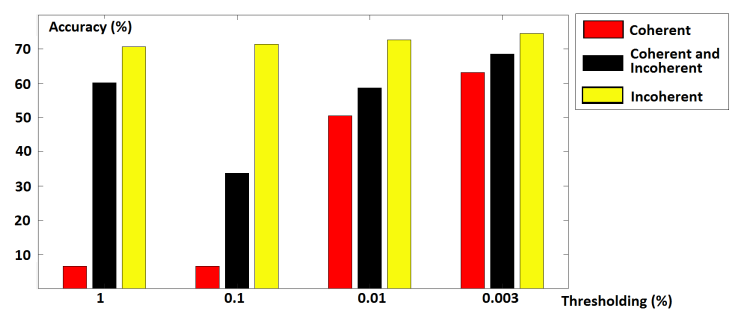

Figure 6: Results from LBP Coherent/Incoherent for some threshold values in the 15-category scenes.

contribution by coherent pixels in the joined histogram. Nonetheless, the coherent pixels have low descriptive power at this moment, and it generates a reduction of the joined histogram.

As the threshold decreases, it increases the amount of coherent regions and more descriptive is the coherent histogram. It also causes a higher descriptive power in joined histogram causing betters outcomes.

In this experiment, the Incoherent histogram reached better results than coherent and joined histograms in all values for the SCT. It is important to point out that the joined histogram has a descriptive vector dimension two times bigger than Incoherent histogram, and this bigger dimension did not imply better accuracy. In our paper, we set $0.005 \%$ to SCT because this value achieved better results for Incoherent histogram.

Figure 7 shows an example of an image from the 15-category scenes for distinct SCT values, the red points represent the coherent pixels. The lower the threshold, the higher the amount of these pixels. Even with the SCT equal to $0.003 \%$, where many coherent regions composed the image, the Incoherent histogram achieved better results.

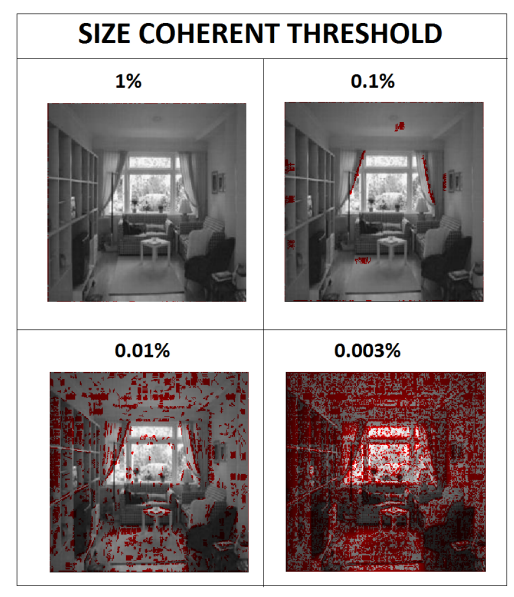

Figure 7: Coherent pixels in image for distinct $\mathrm{SCT}$ values. 


\subsection{Results and Discussion}

In the experiments, we constructed the Incoherent histogram with two buckets, 256 and 128, represented in the name of the descriptor. For example, the vector for histogram of incoherent pixels in LBP image with bucket 256 was called LBPIncoherent-256. As we discarded the bits 0 and 255, the length of LBPIncoherent256 and LBPIncoherent-128 are 254 and 128 respectively, and the CMLBPIncoherent-256 and CMLBPIncoherent-128 are 508 and 256 respectively. For simplicity, in the following part of the text, we refer Centrist and CMCT as the original algorithms. We perform the tests as described below.

\subsubsection{8-category scenes}

The Table 1 compares the classification performance of the proposed method on this dataset. We achieved $80.70 \% \pm 1.0$ accuracy. As already expected, the gist descriptor achieved the best result, since this descriptor is more suitable for scenes with few objects and spatial layout configuration of outdoor scenes (Oliva and Torralba, 2001). The CMLBPIncoherent-256 reached better results than CMCT, the LBPIncoherent256 and LBPIncoherent-128 overcome Centrist. It is worth mentioning that LBPIncoherent-128 dimension is two times smaller than the Centrist dimension.

Table 1: Experimental results for 8-category scenes dataset.

\begin{tabular}{|c||c|}
\hline Feature descriptor & Accuracy \\
\hline Gist & $\mathbf{8 3 . 7 0 \%} \pm \mathbf{0 . 7}$ \\
CMLBPIncoherent-256 & $80.70 \% \pm 1.0$ \\
CMCT & $79.91 \% \pm 1.0$ \\
CMLBPIncoherent-128 & $78.51 \% \pm 1.2$ \\
LBPIncoherent-256 & $77.92 \% \pm 0.4$ \\
MCT8 & $77.07 \% \pm 0.7$ \\
LBPIncoherent-128 & $76.86 \% \pm 0.4$ \\
Centrist & $76.49 \% \pm 0.8$ \\
\hline
\end{tabular}

\subsubsection{5-category scenes}

Table 2 presents the results of the 15-category scenes dataset. In Liu et al. (2011), a method for scene categorization by integrating contextual information region into the bag-of-words approach was proposed, the Region Contextual of Visual Words (RCVW). CMLBPIncoherent overcame this method. The Gist has not been successful, because this dataset contains more indoors scenes than 8-category scenes. Unlike the previous experiment, the CMCT outperformed
CMLBPIncoherent, and the Centrist also overcame the LBPIncoherent. It was due to low recognition rate in class Industrial mainly. While in the CMLBPIncoherent-256 the recognition was $0.56 \%$, for the CMCT was $0.69 \%$. The LBPIncoherent also achieved lower results than Centrist for this class.

Table 2: Experimental results for 15-category dataset.

\begin{tabular}{|c||c|}
\hline Feature descriptor & Accuracy $(\%)$ \\
\hline CMCT & $\mathbf{7 6 . 8 7 \%} \pm \mathbf{0 , 6}$ \\
CMLBPIncoherent-256 & $76.35 \% \pm 0,9$ \\
CMLBPIncoherent-128 & $74.93 \% \pm 0,5$ \\
RCVW & $74.5 \%$ \\
MCT8 & $73.71 \% \pm 0.8$ \\
Centrist & $73.29 \% \pm 1.0$ \\
Gist & $73.28 \% \pm 0.7$ \\
LBPIncoherent-256 & $72.16 \% \pm 0.4$ \\
LBPIncoherent-128 & $71.52 \% \pm 0.9$ \\
\hline
\end{tabular}

\subsubsection{8-class sports events}

Table 3 describes the classification performance of the proposed method on the 8-class sports dataset. In Li and Fei-Fei (2007), the algorithm for classification is a result of global and local features through the scene environment classification and object categorization, the accuracy was $73.4 \%$. However, it is worth point out that the computational cost of this technique is bigger than our method proposed. The CMLBPIncoherent achieved a better result than CMCT, and the LBPIncoherent outperformed Centrist and CMCT. The LBPIncoherent-128, even with four times smaller dimension, achieved a better result than CMCT.

Table 3: Experimental results for 8-class sports dataset.

\begin{tabular}{|c||c|}
\hline Feature descriptor & Accuracy (\%) \\
\hline (Li and Fei-Fei, 2007) & $\mathbf{7 3 . 4 \%}$ \\
CMLBPIncoherent-256 & $72.38 \% \pm 1.1$ \\
CMLBPIncoherent-128 & $70.57 \% \pm 0.9$ \\
LBPIncoherent-256 & $69.06 \% \pm 1.7$ \\
LBPIncoherent-128 & $68.77 \% \pm 1.5$ \\
CMCT & $67.41 \% \pm 1.1$ \\
Centrist & $63.91 \% \pm 2.4$ \\
\hline
\end{tabular}

\subsubsection{7-class indoor scenes}

In the 67 -class indoor scene the gist obtained $21 \%$, the worst result. The CMLBPIncoherent achieved 
the best result with $26.90 \%$. For the indoor scenes, as this dataset, the incoherent technique showed better results than techniques with more level of processing as the technique proposed by $\mathrm{Li}$ and Fei-Fei (2007).

Table 4: Experimental results for 67-class indoor scenes.

\begin{tabular}{|c||c|}
\hline Feature descriptor & Accuracy (\%) \\
\hline CMLBPIncoherent-256 & $\mathbf{2 7 . 7 0 \%} \pm \mathbf{0 . 6}$ \\
CMLBPIncoherent-128 & $26.64 \% \pm 1.9$ \\
CMCT & $25.82 \% \pm 0.7$ \\
Li and Fei-Fei (2007) & $25 \%$ \\
LBPIncoherent-256 & $23.04 \% \pm 1.2$ \\
Centrist & $22.46 \% \pm 0.8$ \\
Gist & $21 \%$ \\
LBPIncoherent-128 & $22.27 \% \pm 0.7$ \\
\hline
\end{tabular}

\section{Conclusion}

In this paper, we proposed the CMLBPIncoherent, a visual descriptor that captures structural properties by calculating the histogram of the LBP image. Also, the CMLBPIncoherent combines contextual information of local structures and discards homogeneous regions. As the LBP, this method has some advantages as the low amount of parameters to tune and low computational cost.

The histogram of the incoherent information overcame the coherent information and the histogram generated when the both information are concatenated. Therefore, the homogeneous regions represented by coherent information disturbs the scene recognition and is not appropriate to our descriptor visual. The recognition achieved better results when the threshold of the size of homogeneous regions had lower values, approximately $0.005 \%$ of image size.

Through the experiments carried out, the CMLBPIncoherent achieved better results than CMCT and Centrist, in general. In some cases, even with four times smaller dimension, the LBPIncoherent achieved better results than CMCT.

The CMLBPIncoherent showed good results both indoors and outdoors scenes dataset, unlike other algorithms that are more specific for the only type of scene layout.

The contextual information from CMLBPIncoherent improved the power description of the image representation, since its results achieved better performance than the LBPIncoherent.

In our future research, we intend to use spatial layout information, separating the image in grids, to aggregate local approach in our method.
We also intend to test color information in some datasets to improve the results.

\section{Acknowledgment}

The authors would like to thanks the Coordenação de Aperfeiçoamento de Pessoal de Nível Superior (CAPES) for the scholarship granted.

\section{References}

Bian, X., Chen, C., Tian, L. and Du, Q. (2017). Fusing local and global features for highresolution scene classification, IEEE Journal of Selected Topics in Applied Earth Observations and Remote Sensing $\mathbf{P P}(99)$ : 1-13.

Burges, C. J. (1998). A tutorial on support vector machines for pattern recognition, Data mining and knowledge discovery 2(2): 121-167.

Chang, C.-C. and Lin, C.-J. (2011). Libsvm: a library for support vector machines, ACM Transactions on Intelligent Systems and Technology (TIST) 2(3): 27.

Chen, C., Zhou, L., Guo, J., Li, W., Su, H. and Guo, F. (2015). Gabor-filtering-based completed local binary patterns for landuse scene classification, Multimedia Big Data (BigMM), 2015 IEEE International Conference on, IEEE, pp. 324-329.

Chu, J. and Zhao, G.-H. (2014). Scene classification based on sift combined with gist, Information Science, Electronics and Electrical Engineering (ISEEE), 2014 International Conference on, Vol. 1, IEEE, pp. 331-336.

de Souza Gazolli, K. and Teatini Salles, E. O. (2014). Exploring neighborhood and spatial information for improving scene classification, Pattern Recognit. Lett. 46: 83-88.

Fan, Y. (2016). Improved scene classification using region semantics and spatial context information, 2016 19th International Conference on Computer and Information Technology (ICCIT), pp. 443-450.

Fei-Fei, L. and Perona, P. (2005). A bayesian hierarchical model for learning natural scene categories, Computer Vision and Pattern Recognition, 2005. CVPR 2005. IEEE Computer Society Conference on, Vol. 2, IEEE, pp. 524531.

Jiang, A., Wang, C., Xiao, B. and Dai, R. (2010). A new biologically inspired feature for scene image classification, Pattern Recognition (ICPR), 2010 20th International Conference on, IEEE, pp. 758-761. 
Lazebnik, S., Schmid, C. and Ponce, J. (2006). Beyond bags of features: Spatial pyramid matching for recognizing natural scene categories, Computer vision and pattern recognition, 2006 IEEE computer society conference on, Vol. 2, IEEE, pp. 2169-2178.

Li, L.-J. and Fei-Fei, L. (2007). What, where and who? classifying events by scene and object recognition, Computer Vision, $200 \%$. ICCV 200\%. IEEE 11th International Conference on, IEEE, pp. 1-8.

Liu, S., Xu, D. and Feng, S. (2011). Region contextual visual words for scene categorization, Expert Systems with Applications 38(9): 11591-11597.

Mandhala, V. N., Sujatha, V. and Devi, B. R. (2014). Scene classification using support vector machines, Advanced Communication Control and Computing Technologies (ICACCCT), 2014 International Conference on, IEEE, pp. 1807-1810.

Ojala, T., Pietikäinen, M. and Harwood, D. (1996). A comparative study of texture measures with classification based on featured distributions, Pattern recognition 29(1): 5159.

Oliva, A. and Torralba, A. (2001). Modeling the shape of the scene: A holistic representation of the spatial envelope, International journal of computer vision 42(3): 145-175.

Pass, G., Zabih, R. and Miller, J. (1997). Comparing images using color coherence vectors, Proceedings of the fourth ACM international conference on Multimedia, ACM, pp. 65-73.

Potter, M. C. (1975). Meaning in visual search, Science 187(4180): 965-966.

Quattoni, A. and Torralba, A. (2009). Recognizing indoor scenes, Computer Vision and Pattern Recognition, 2009. CVPR 2009. IEEE Conference on, IEEE, pp. 413-420.

Salyut, J. and Kurnaz, C. (2018). Profile face recognition using local binary patterns with artificial neural network, 2018 International Conference on Artificial Intelligence and Data Processing (IDAP), pp. 1-4.

Shahriari, M. and Bergevin, R. (2017). Land-use scene classification: a comparative study on bag of visual word framework, Multimedia Tools and Applications pp. 1-17.

URL: $\quad$ http://dx.doi.org/10.1007/s11042016-4316- $z$
Shi, J., Li, X. and Dong, Y. (2015). How to represent scenes for classification?, Signal and Information Processing (ChinaSIP), 2015 IEEE China Summit and International Conference on, IEEE, pp. 191-195.

Tabatabaei, S. M. and Chalechale, A. (2018). One dimensional second order derivative local binary pattern for hand gestures classification using semg signals, 2018 8th International Conference on Computer and Knowledge Engineering (ICCKE), pp. 16-19.

TURAN, E., UÃ ĞAR, F. and DANDIL, B. (2018). Local binary patterns and extreme learning machine based texture classification of marbles, 2018 International Conference on Artificial Intelligence and Data Processing (IDAP), pp. 1-6.

Vailaya, A., Jain, A. and Zhang, H. J. (1998). On image classification: City images vs. landscapes, Pattern Recognition 31(12): 19211935.

Wu, J. and Rehg, J. M. (2011). Centrist: A visual descriptor for scene categorization, IEEE transactions on pattern analysis and machine intelligence 33(8): 1489-1501.

Yang, J., Jiang, Y.-G., Hauptmann, A. G. and Ngo, C.-W. (2007). Evaluating bag-of-visualwords representations in scene classification, Proceedings of the international workshop on Workshop on multimedia information retrieval, ACM, pp. 197-206.

Zabih, R. and Woodfill, J. (1994). Non-parametric local transforms for computing visual correspondence, European conference on computer vision, Springer, pp. 151-158. 